\title{
La anorexia nerviosa y la post modernidade
}

Conferência apresentada em 10 de setembro de 2015 na Faculdade de Ciências Médicas da Santa Casa de São Paulo.

Otto Dörr Zegers ${ }^{1}$

¿Cuál es la relación entre la anorexia nerviosa y la post modernidad, fuera del hecho que ella aparece recién en la modernidad tardía y en el comienzo de la época que estamos viviendo? Para responder esta pregunta tendríamos que revisar algunas de las características de nuestro tiempo, que a nuestro parecer pudieran hacer comprensible el surgimiento en él de esta misteriosa enfermedad. Estas son: el imperio de la técnica, la obscenidad y la pérdida del sentido religioso de la existencia.

Uno de los rasgos fundamentales de la post modernidad y que también caracteriza al período anterior, el de la modernidad, es el imperio de la técnica. En la modernidad el extraordinario desarrollo adquirido por las ciencias naturales llevó a la creación de instrumentos cada vez más complejos que han facilitado mucho la vida humana y que han hecho al hombre progresivamente más independiente de su medio. Los ejemplos son casi infinitos: las máquinas a vapor, la electricidad, los motores de combustión, el telégrafo, el teléfono, los aparatos de reproducción del sonido y de la imagen, etc. En la post modernidad esto llega al paroxismo con el invento de la computación y todos sus derivados, a través de los cuales se ha logrado, en cierto modo, la absoluta inmediatez y la total ubicuidad, vale decir, la superación del tiempo y del espacio. La consecuencia final ha sido el advenimiento de un mundo cada dia más virtual. Sobre los peligros de la técnica nos han advertido con mucha lucidez el filósofo Martin Heidegger y el poeta Rainer Maria Rilke. Asi, en su ensayo "La pregunta por la técnica”, Heidegger nos dice: "El desocultar que domina a la técnica moderna tiene el carácter... de la pro-vocación. Ésta acontece de tal manera que se descubren las energías ocultas en la naturaleza; lo descubierto es transformado; lo transformado, acumulado; lo acumulado, a su vez, repartido y lo repartido se renueva cambiado." (1983, p. 83). Rilke, por su parte, denuncia el poder y el carácter enajenante de la técnica en el Soneto $\mathrm{N}^{\circ}$ XVIII de la Primera Parte de los Sonetos a Orfeo:

${ }^{1}$ Universidad de Chile e de la Universidad Diego Portales. 
¿Escuchas, Señor, a lo nuevo

resonar y temblar?

A ensalzarlo

vienen los heraldos.

Verdad es que ningún oído

está del estrépito a salvo,

pero el mundo de la máquina

quiere ser alabado ahora.

Mira, la máquina:

cómo se revuelca y se venga,

cómo nos deforma y agobia.

Pero aunque tenga de nosotros la fuerza,

que ella, sin pasión,

sirva y funcione."

"Lo nuevo" representa por cierto la revolución tecnológica, ensalzada por todos desde mediados del siglo XIX. Esa glorificación de la ciencia y del progreso ("A ensalzarlo / vienen los heraldos") ha persistido por décadas, mientras las dudas planteadas por la misma física moderna se olvidan en la embriaguez producida por los continuos avances tecnológicos. Para Rilke este mundo de las máquinas y su poder sin límite aparece ligado esencialmente al ruido, a la ausencia de silencio y, por ende, de paz. Así, en la primera estrofa "lo nuevo" se presenta como aquello que "resuena y tiembla" y en la segunda el poeta nos recuerda que nadie está "a salvo del estrépito". El hombre no tiene donde esconderse del ruido en el mundo moderno y éste es producido casi exclusivamente por las máquinas. En el primer terceto el poeta nos advierte con duras palabras sobre el peligro que significa la técnica para el hombre, pues la máquina terminará "vengándose" de nosotros, porque "nos deformará y nos agobiará". Con respecto a la primera consecuencia de esta venganza, bastaría pensar en la preocupante deformación de la mente de jóvenes y niños producida por la televisión y la computación. El "agobio" podría identificarse con el impresionante aumento de los 
cuadros depresivos durante los últimos 50 años. En el último terceto el poeta acepta el hecho que las máquinas han sido inventadas libremente por el hombre (tienen "de nosotros la fuerza") y que nos han facilitado la vida en muchos aspectos ("que ella... sirva y funcione"), pero al mismo tiempo nos pide no olvidar que ellas trabajan "sin pasión", vale decir, que al carecer de sentimientos es muy fácil que se transformen en instrumentos de destrucción y depredación.

¿Pero qué es lo que subyace a este imperio de la técnica? En nuestra opinión, la técnica moderna ha sobrepasado el impulso natural del hombre a construir instrumentos, sobre la base de los materiales que ofrece la naturaleza. Y este afán constructor, que a primera vista parece tan positivo, ha terminado transformando la realidad en una imagen, en un artificio que llega al extremo de la virtualidad. Heidegger dice al respecto una frase memorable: "La transformación de la realidad en su imagen, eso es la modernidad". Rilke también había anunciado el imperio de la imagen, cuando dice en la Séptima Elegía del Duino:

"En ningún sitio, amada, habrá mundo si no es dentro...

pues lo externo, siempre más insignificante, se desvanece. Donde hubo una vez una casa duradera, oblicua irrumpe ahora una figura imaginada, algo que pertenece por entero a lo pensable, como si estuviera aún del todo en el cerebro. Vastas reservas de energía se crea el espíritu del tiempo...”, etc.

El verdadero mundo se encuentra en la profundidad del espíritu humano. El mundo externo, dominado por el sentido de la vista, va perdiendo su verdadero significado. Todo aquello que fue importante y duradero para el hombre, como una casa, por ejemplo, empieza a ser reemplazado por su imagen, por estructuras cada vez más abstractas y artificiales (“algo que pertenece por entero a lo pensable”). De paso el poeta nos advierte sobre la necesaria, pero peligrosa acumulación de energía en la modernidad, lo que bien se podría considerar como una premonición de las futuras centrales atómicas. 
La anoréctica sería un ejemplo extremo de este predominio de la imagen sobre la realidad. Y decimos extremo, porque no es ya el espacio, ni el tiempo, ni los objetos que nos rodean, los que han sido transformados en artificios, sino el mismo cuerpo vale decir, lo más próximo y propio que tenemos. En la anoréctica el cuerpo deviene una "cosa" separada del yo mismo, el cual deja entonces de ser un sujeto encarnado. El cuerpo es cosificado y adquiere el carácter de un mero ob-jeto, cuyo total valor estaría en la apariencia del diseño, como si fuese un utensilio a contemplar, dimensionado como pura imagen pública. La anoréctica sufre una alienación de su propio cuerpo, que experimenta no solo como in-apropiado, sino como un cuerpo enajenado, un cuerpootro, que es externo a su dimensión personal, un cuerpo que coloca su intimidad al alcance de la humillante y aniquiladora mirada del otro. Esto es lo que la lleva a intentar establecer un continuo y total control sobre este su cuerpo enemigo (ver Pelegrina, 1999).

Así como la post modernidad ha manipulado la realidad a través de la técnica hasta el punto de hacerla casi del todo artificial, la anoréctica intenta construirse un cuerpo sin volumen, un cuerpo también en cierto modo virtual, un cuerpo que no ocupa espacio y que no siente, ni siquiera los instintos. La anoréctica piensa que si logra controlar su cuerpo, sobre todo su apetito, se será suficientemente poderosa para controlar todo lo demás. Este intento "fabril" de fabricar su cuerpo a discreción corresponde muy bien a la actual pretensión de tener una "vida de diseño". El sociólogo Giddens afirma respecto a la postmodemidad: "Lo que puede aparecer como un culto narcisista de la apariencia corporal es de hecho la expresión de una preocupación mucho más profunda por “construir el cuerpo". Así es como esta manipulación del cuerpo se da también en otras expresiones de la post modernidad, como por ejemplo en el aumento exponencial de las operaciones de cirugía plástica y de los tatuajes. Un ejemplo dramático de lo mismo es el del famoso cantante Michael Jackson. Estas conductas tienen en común con la anorexia nerviosa el tomar al cuerpo como objeto y la excesiva importancia que se da a la apariencia, a eso que se muestra, pero se diferencian en el hecho no menor que la anoréctica se juega la vida en su empeño ilusorio de hacer desaparecer su cuerpo-objeto y transformarse en puro espíritu.

Quisiera mencionar uma segunda característica de la post modernidad que está íntimamente relacionada com la anorexia nerviosa. Me refiero a la obscenidad. 
No se trata sólo de la tendencia progresiva a eliminar toda forma de pudor, algo que aparece día a día bajo la forma de nudismo, de propaganda que, para seducir, emplea cuerpos o escenas en el límite de lo pornográfico, de esas películas explícitamente pornográficas que se muestran en el cine, la televisión o la Internet. Se trata más bien de que nuestro mundo, tan dominado por el sentido de la vista, busca desesperadamente el mirar los objetos hasta en su más ínfimo detalle, desde todas las perspectivas posibles, repitiendo la contemplación una y otra vez, por medio de la filmadora, el aparato de video, o más recientemente, del teléfono celular. La obscenidad comienza cuando ya no hay ni ilusión ni fantasía, cuando todo se ha hecho inmediatamente transparente y visible, sometido a la cruda e inexorable luz de la información y de la comunicación. El filósofo francés Jean Baudrillard (1988) ha descrito este fenómeno en forma insuperable: "En muchos casos nuestra imaginería erótica y pornográfica, toda esa panoplia de senos, nalgas y sexos, no tiene más sentido que éste: expresar la inútil objetividad de las cosas. La desnudez sólo sirve como intento desesperado para subrayar la existencia de algo... Lo sexual no es más que un ritual de la transparencia. Antes había que esconderlo; hoy en cambio sirve para esconder la raquítica realidad.” (p. 27).

Nada puede ser más obsceno que el cuerpo de la anoréctica. Y es obsceno tanto cuando se muestra como cuando se oculta. Es sabido que algunas pacientes gustan de ocultar su cuerpo con ropas amplias, mientras otras o las mismas en otro momento, experimentan placer escandalizando a los demás mostrándoles sus huesos. Pero en ambos casos, ya sea cuando ocultan su cuerpo con enormes ropones o cuando lo muestran descarnado, hay un estar en escena, hay un predominio de la apariencia, un imperio de la imagen, vale decir, ob-scenidad. Recordemos que "ob" en latín significa estar ahí al frente, a la vista y "scenidad" deriva naturalmente de escena. La anoréctica comparte esa obscenidad con el obeso y quizá algo más aún, el intento de hacer desaparecer el cuerpo. Esto lo expresa magníficamente el mismo Jean Baudrillard (1987) al referirse a la abundancia de obesos en los Estados Unidos: "No es la obesidad compensatoria del subdesarrollo, ni la alimentícia del subalimentado. Paradójicamente es un modo de desaparición del cuerpo. La regla secreta que delimita la esfera del cuerpo ha desaparecido. La forma secreta del espejo, por la que el cuerpo cuida de sí mismo y de su imagen, ha sido abolida y sustituida por la redundancia irrefrenable de un organismo viviente. Ya no hay límite, ya no hay trascendencia: es como si el cuerpo ya no se 
opusiera a un mundo exterior, sino que intentara digerir el espacio en su propia apariencia". Y termina diciendo algo que no tiene que ver con nuestro tema, pero que es sumamente ingenioso: "Estos obesos son fascinantes por su olvido total de la seducción" (p. 27).

Otra forma de expresarse esta obscenidad postmodema, que tanto tiene que ver con nuestras anorécticas, es el progresivo debilitamiento de esa gran conquista humana que es la separación entre el espacio público y el espacio privado. El hombre contemporáneo vive la proximidad absoluta, la instantaneidad total de las cosas, la sensación de que no hay defensa ni retirada ni privacidad posible. Es el fin de la interioridad y de la intimidad. Es la excesiva exposición y transparencia del mundo y de sí mismo. Como dice el mismo Baudrillard: "El hombre ya no es capaz de producir los límites de su propio ser”. Y la anoréctica es la consecuencia más extrema de esta situación, pues ella no puede deslindar su intimidad del espacio público, porque lo percibe como perteneciente a los otros. Ella se siente presa del espacio exterior y vive su propio cuerpo como un objeto expuesto y que ha arrastrado consigo a su intimidad subjetiva.

Un último rasgo de la post modernidad que quisiera destacar en el día de hoy es la pérdida del sentido religioso de la existencia. Esa re-ligazón fundamental del hombre con lo divino y que lo sostuvo por siglos y milenios ha sido reemplazada primero por la diosa razón, después por la ciencia y en las últimas décadas, por el dinero y el placer, tomado este último en su sentido más efímero y decadente. Por cierto que la pérdida del lenguaje tiene que ir aparejada necesariamente con la pérdida del sentido religioso, porque toda religión es revelación y por lo tanto, verbo. Y Dios mismo ha sido definido desde San Juan como el logos, el verbo por antonomasia. También el esfumarse del passado y del futuro, tan proprio de la actitud hedonista actual, ha hecho insostenible uma auténtica vida religiosa, por cuanto ésta requiere tanto del relato sagrado, del mito que ilumina y ayuda (que viene del pasado), como de la esperanza en una vida más allá de la muerte, que dé sentido a nuestra vida terrena (el futuro). Muy relacionado con lo mismo está el olvido del dolor y del sufrimiento, que desde siempre han constituido la via regia para acceder a la dimensión trascendente. El dolor ha sido anestesiado y el sufrimiento, negado por los tranquilizantes, los antidepresivos o las drogas. Rilke, como 
en tantas otras cosas, se adelantó a los tiempos y denunció este fenómeno ya en 1922, cuando en su famoso Soneto $N^{\circ}$ XIX de la Primera Parte de los Sonetos a Orfeo, dice:

\author{
No se ha reconocido el dolor \\ ni se ha aprendido el amor \\ y lo que nos aleja en la muerte
}

no ha sido develado aún.

Sólo el canto sobre la tierra

santifica y celebra.

Sin duda que hemos olvidado el dolor, ya no se ama como en otros tiempos y la muerte ha sido simplemente negada. Pero frente a esos tres elementos del drama de nuestra época: no reconocer el dolor, no aprender el amor y no aceptar la muerte, el poeta nos muestra un camino: "Sólo el canto sobre la tierra / santifica y celebra". En el canto se unen la palabra y la música. La palabra es, según Heidegger (1959), "la morada del ser", pero al mismo tempo el puente que une la tierra y el cielo, los mortales y los dioses, mientras la música - el más elevado producto del espíritu humano - nacía, según el mismo Rilke, como estela de vibraciones dejada por la muerte del semi-dios Lino, otro hijo de Apolo, en medio del vacío del espacio primigenio, “vibración que aún ahora nos arrebata, nos consuela y nos ayuda" (final de la Primera Elegía, 2000, p. 33). Reproduciré aquí algunas frases de la interpretación que hace Heidegger de estos versos: “... los mortales apenas son capaces de reconocer su propia mortalidad... la muerte se ha retirado hacia lo enigmático. El misterio del dolor permanece oculto. No se ha aprendido el amor, pero los mortales son en la medida que existe el linguaje, porque todavía el canto prevalece sobre la tierra precaria y la voz del que canta mantiene todavía abierta la huella de lo sagrado... ha desaparecido aquel ámbito essencial en el cual co-pertenecen el dolor, el amor y la muerte. Hay ocultamiento en la medida que el ámbito de esta co-pertenencia es el abismo del ser. Pero todavía queda el canto que llama a la tierra. ¿Qué es el canto mismo? ¿Cómo se hace un mortal capaz de cantar? ¿Desde dónde canta el canto? ¿Hasta donde alcanza el canto en el abismo?” (1980, p. 265). 
Pienso que las anorécticas representan un ejemplo extremo de esta pérdida del sentido religioso que caracteriza a nuestra época. Ellas viven en la pura inmanencia, en lo inmediato mismo de esa prisión que es el cuerpo objeto e intentando hacerlo desaparecer. Ninguna otra cosa les interesa fuera de dominarlo o de ocuparse, paradójicamente, con el tema del alimento, que es justo lo que ellas odian y aman a la vez. Pasan horas deshaciendo un trozo de pan que lo ingieren miga a miga, o mirando y aún obligando a los demás a comer para mirarlos en ese acto cual voyeurs. No se reconoce el dolor propio ni el ajeno. Ellas no sienten nada, ni siquiera el dolor de las contracciones del hambre. Tampoco perciben el dolor de sus familiares frente a su enfermedad. La muerte es simplemente negada. Es impresionante la indiferencia con que toman la proximidad de la muerte cuando han alcanzado grados extremos o irrecuperables de desnutrición. Tampoco el amor les interesa, aunque son muy dependientes de la madre. Es sabido que la mayor parte de ellas no se casa ni tiene pareja. Es un mundo que desconoce el amor, el olvido de sí mismo y para qué decir, la búsqueda de un sentido trascendente. Y por esta razón no pudo haber anorécticas en la Edad Media ni en el Renacimiento ni menos aún en el Romanticismo. En esos siglos y también durante todos los anteriores, el ser humano se vivió a sí mismo como acogido en los brazos de Dios y todo tenía un sentido desde la Providencia divina: el nacimiento, la enfermedad y la muerte, tanto las alegrías como los dolores y el cuerpo no solo tenía un valor en sí mismo, sino que era sagrado, sacralidad que alcanzaba su mayor altura en el sacramento de la Comunión. El cuerpo de la anoréctica, en cambio, es lo contrario del cuerpo sagrado y se encuentra muy próximo al cuerpo obsceno de la post modernidad, ese que tan bien describiera Jean Baudrillard con las siguientes palabras: “... (este cuerpo), al ser objeto de esa mirada obscena que todo lo desnuda, pierde su dignidad y se transforma en una diversidad de superficies, en una pululación de múltiples objetos, donde se perde su finitud y su seducción: cuerpo metastático y fractal, ya no llamado a ninguna forma de resurrección" (op. cit., p. 38).

¿Y qué ocurre con las bulímicas? ¿Por qué aparecen en la postmodernidad misma, cien años después de las anorécticas? Ambas tienen que ver con el tema del alimento, pero son muy diferentes. En primer lugar, porque las bulímicas no se juegan la vida en su preocupación excesiva por el cuerpo. Tampoco comparten el ideal ascético ni tratan de controlar su cuerpo y el entorno como lo hacen las anorécticas. Asimismo, ellas no 
rechazan y/o niegan la sexualidad. De aspecto son mucho más normales que las otras y no solo por el enflaquecimiento extremo de unas y las alzas y bajas moderadas de peso de las otras. Tanto su presentación como su personalidad pasan en general desapercibidas, mientras que las anorécticas llaman de inmediato la atención. Pienso que tampoco ese lado fabril, de fabricar su cuerpo al modo de un instrumento, tan propio de las anorécticas, corresponda a la relación que la bulímica tiene con el suyo. Con respecto a esas tres características de la modernidad mencionadas en el poema de Rilke: no reconocer el dolor, no aprender el amor y alejar o negar la muerte, tampoco pareciera la paciente bulímica identificarse con ellas. ¿Dónde estaría entonces el vínculo entre la bulimia y la postmodemidad? Creo que el problema radica en la identidad y su profunda crisis en nuestra época. Primero un hecho de observación: en mi larga experiencia con este tipo de pacientes he podido observar la frecuente asociación entre esta conducta y la llamada personalidad limítrofe. Esta personalidad es también un producto postmodemo - las primeras descripciones de ella prácticamente coinciden con las de la bulimia - y está caracterizada por, a nivel sintomático, gran inestabilidad afectiva, impulsividad, relaciones interpersonales que oscilan permanentemente entre la idealización y la devaluación, sentimientos de rabia inapropiados, intolerancia a la frustración, sensación de vacío y un serio problema a nivel de la identidad. Sus continuos cortes em los brazos y el uso casi regular de tatuajes y piercings han sido interpretados - y con razón - como una forma de búsqueda de sí mismo, de llenar ese vacío existencial que experimentan.

La relación entre la personalidad limítrofe y la postmodemidad ha sido desarrollada recientemente por Anneliese Dörr (2012). En la época pre-moderna la identidad estaba dada por la tradición, la familia, la escuela, la religión. En la modernidad se empieza a producir un cierto grado de independencia con respecto a la tradición y la identidad se logra también, preferentemente a través del esfuerzo personal. Es el "self made man" del mundo norteamericano de la primera mitad del siglo XX. Ambas sociedades tenían en común la cultura o psicología de la escasez, de ahí la necesidad del esfuerzo mancomunado con todos los familiares, por ejemplo, o personal. En la modernidad tardía o postmodernidad, en cambio, la psicología de la escasez es reemplazada por una psicología de la abundancia, cuya consecuencia casi necesaria es el consumo descontrolado. La identidad empieza a ser dirigida por los medios de comunicación, por 
los grupos de pertenencia (las tribus urbanas), en último término, la identidad va a depender de la capacidad de consumo y de la aprobación de los otros. Este contexto sociológico estaria generando esta particular "difusión de la identidad" tan propia de las personalidades limítrofes. Las instituciones tradicionales están cuestionadas o han fracasado y sus normas están siendo substituidas por pautas orientadas desde las redes sociales y las ofertas del mercado. Los más débiles, absorbidos por el mercado de la imagen y sin capacidad para aferrarse a valores permanentes, van dejándose llevar de una imagen a otra, sin un sentido que les permita articular de manera coherente sus experiencias de vida. Se requieren muchos más recursos personales para enfrentar esta sociedad compleja, cambiante y muchas veces hostil.

Las muchachas bulímicas constituirían una particular versión de este drama de la juventud postmodema. Para ellas sí vale el tema del ideal de delgadez que muestran las modelos y que quieren alcanzar en forma rápida y fácil. A algunas les cuesta mantenerse delgadas, porque son de naturaleza un poco más corpulentas. Otras, porque desde su impulsividad y la búsqueda de la satisfacción inmediata no pueden controlar el mayor apetito propio de la juventud, hasta que descubren el vómito como una forma si no de lograr esa imagem ideal, al menos acercarse a ella. Y ahí empieza el círculo vicioso que puede llegar a transformasse en un infierno: el vacío de su existencia sin valores ni ideales fuera de la imagen y el consumo las impulsa a los atracones. Como no pueden soportar la idea de subir de peso, vomitan. Pero la sensación que experimentan después del vómito es de mayor vacío y frustración que la que tenían antes de primer episodio de hiperfagia, lo que las lleva al siguiente atracón y así sucesivamente. Lo más llamativo en estas muchachas es este estrechamiento de su existencia en torno al tema del peso y la figura. Y así como los jóvenes limítrofes buscan su identidad a través de las drogas y las automutilaciones, las jóvenes bulímicas, compartiendo el mismo vacío con aquellos, buscan la suya en este frenético intento de alcanzar esa figura delgada que exige el mercado de la imagen y el consumo. El caso de las anorécticas, como vimos, es mucho más complejo. No es la delgadez lo que ellas buscan, como las bulímicas, no es ese cuerpo ideal que muestran las revistas. Lo que ellas quieren es no tener cuerpo, no ocupar volumen en el espacio, ser puro espíritu, pero un espíritu que no está abierto hacia el otro, hacia la belleza o hacia la sabiduría, sino solo referido a sí mismo. De ahí su soledad, su soledad cósmica. 